\title{
Erasing the Past and Impacting the Future with Kaons at a Phi-Factory
}

\author{
Beatrix C. Hiesmayr ${ }^{* \dagger}$ \\ Faculty of Physics, University of Vienna, Boltzmanngasse 5, 1090 Vienna, Austria \\ E-mail: Beatrix.Hiesmayreunivie.ac.at
}

\section{Albert Bramon}

Grup de Física Teòrica, Universitat Autònoma de Barcelona, E-08193 Bellaterra, Spain

\section{Antonio Di Domenico}

Dipartimento di Fisica, Universita' di Roma "La Sapienza", and INFN Sezione di Roma, I-00185 Rome, Italy

\section{Gianni Garbarino}

Dipartimento di Fisica Teorica, Università di Torino and INFN, Sezione di Torino, I-10125

Torino, Italy

\section{Apollo Go}

Physics Department, National Central University, 32001 Chung-Li, Taiwan

\begin{abstract}
The well known quantum eraser concepts are applied to entangled kaons. Due to different measurement procedures, a specific feature of neutral kaons not shared by other quantum systems, we show that novel options for the quantum eraser phenomena open. Moreover, the proposed kaonic erasers may in future be experimentally demonstrated with the upgraded KLOE detector at the $\phi$-factory.
\end{abstract}

KAON International Conference

May 21-25 2007

Laboratori Nazionali di Frascati dell'INFN, Rome, Italy

\footnotetext{
* Speaker.

$\dagger$ Thanks for the great conference and the opportunity to visit the accelerator.
} 


\section{Introduction}

Two hundred years ago Thomas Young taught us that photons interfere. Nowadays also experiments with very massive particles, like the fullerenes [1], have impressively demonstrated that fundamental feature of quantum mechanics. It seems that there is no physical reason why not even heavier particles should interfere except for technical ones. Then it was shown that the knowledge of the path through the double slit is the reason why interference is lost, however, the gedanken experiment of Scully and Drühl in 1982 [2] shocked the physics community: if the knowledge of the path of the particle is erased, interference is brought back again!

Since that work many different types of quantum erasures have been analyzed and experiments were performed with atom interferometers [3] and entangled photons [4, 5, 6, 7, 8, 9]. Generally, the meter, the quantum system which carries the "mark" of the path taken, is a system spatially separated from the interfering system called the object system. It turned out that the decision to erase or not the mark of the meter system - and therefore to observe or not interference - can be taken long after the measurement on the object system has been completed which is called an eraser in the "delayed choice" mode and captures best the essence and the most subtle aspects of the quantum eraser phenomenon. This was also nicely phrased by Aharonov and Zubairy in their review article [10] as "erasing the past and impacting the future".

Here we want to present four different types of quantum erasure concepts for neutral kaons, proposed in Refs. [11, 12]. Two of them are analogous to erasure experiments performed with entangled photons, e.g. Refs. [4, 5], sketched in Figs. 12, In the first experiment with photons the erasure operation is carried out "actively", i.e., by exerting the free will of the experimenter, whereas in the latter experiment the erasure operation is carried out "partially actively", i.e., the mark of the meter system is erased or not by a well known probabilistic law, e.g., by a beam splitter. However, different to photons the kaons can be measured by an active or a passive procedure (see Sect. 2).

This offers new quantum erasure possibilities which can only be achieved with kaons, a naturally interfering and decaying (marking) system. And in this way proves the very concept of a quantum eraser, namely, sorting events to available information.

We believe that the upgraded KLOE-2 detector at the DA $\phi$ NE machine offers the possibility for an experimental demonstration of the here described "kaonic erasers". Monte Carlo and design studies are in progress.

\section{Two measurement procedures}

For neutral kaons there exist two physical alternative bases, accordingly we have two observables for the kaons, namely the projectors to the two bases. The first basis is the strangeness eigenstate basis $\left\{\left|K^{0}\right\rangle,\left|\bar{K}^{0}\right\rangle\right\}$, it can be measured by inserting along the kaon trajectory a piece of ordinary matter, which corresponds to an active measurement of strangeness. Due to strangeness conservation of the strong interactions the incoming state is projected either onto $K^{0}$ by $K^{0} p \rightarrow K^{+} n$ 


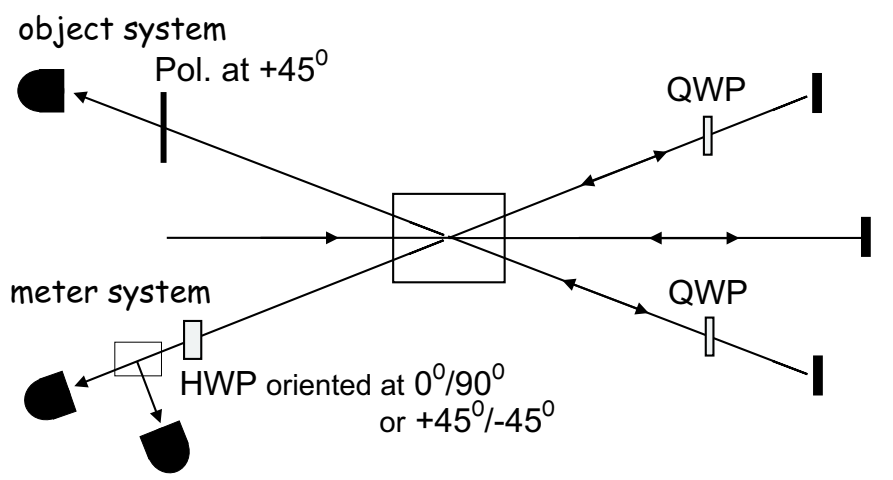

Figure 1: Here the setup for an active eraser is sketched. A pump beam transverses twice a, e.g., type II crystal. The pairs produced in the first passage through the crystal cross two times a quarter-wave plate (QWP) which transforms an original horizontal polarized photon into a vertical one and vice versa. The pairs produced in the second passage through the crystal is directly sent to the measurement devices. The signal (object) photon is always measured after crossing a polarization analyzer aligned at $+45^{\circ}$. The idler (meter) photon crosses a half-wave plate (HWP) oriented at $0^{\circ}, 90^{\circ}$ (first setup) or $\pm 45^{\circ}$ (second setup) and is then analyzed by a polarization beam splitter. In the first setup - meter photon is measured in the $H / V$ basis - one has full which way information, namely if the pair was produced at the first or second passage. In the second setup - meter photon is measured in the $+45^{\circ} /-45^{\circ}$ basis - the information on the first or second passage is erased, one observes fringes or antifringes.

or onto $\bar{K}^{0}$ by $\bar{K}^{0} p \rightarrow \Lambda \pi^{+}, \bar{K}^{0} n \rightarrow \Lambda \pi^{0}$ or $\bar{K}^{0} n \rightarrow K^{-} p$. Here nucleonic matter plays the same role as a two channel analyzer for polarized photon beams.

Alternatively, the strangeness content of neutral kaons can be determined by observing their semileptonic decay modes. The strange quark $s$ decays weakly:

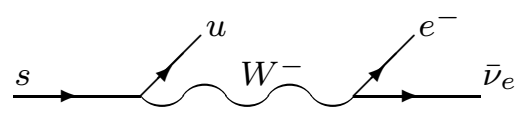

Due to their quark content the kaon $K^{0}(\bar{s} d)$ and the anti-kaon $\bar{K}^{0}(s \bar{d})$ have the following different decays:

$$
\begin{array}{llll}
K^{0}(d \bar{s}) \longrightarrow \pi^{-}(d \bar{u}) l^{+} v_{l} & \text { where } & \bar{s} \longrightarrow \bar{u} l^{+} v_{l} \\
\bar{K}^{0}(\bar{d} s) \longrightarrow \pi^{+}(\bar{d} u) l^{-} \bar{v}_{l} & \text { where } & s \longrightarrow u l^{-} \bar{v}_{l},
\end{array}
$$

with $l$ either muon or electron, $l=\mu, e$. When studying the leptonic charge asymmetry

$$
\delta=\frac{\Gamma\left(K_{L} \rightarrow \pi^{-} l^{+} v_{l}\right)-\Gamma\left(K_{L} \rightarrow \pi^{+} l^{-} \bar{v}_{l}\right)}{\Gamma\left(K_{L} \rightarrow \pi^{-} l^{+} v_{l}\right)+\Gamma\left(K_{L} \rightarrow \pi^{+} l^{-} \bar{v}_{l}\right)}
$$

we notice that $l^{+}$and $l^{-}$tag $K^{0}$ and $\bar{K}^{0}$, respectively, in the $K_{L}$ state, and the leptonic asymmetry (2.2) is expressed by the probabilities $|p|^{2}$ and $|q|^{2}$ of finding a $K^{0}$ and a $\bar{K}^{0}$, respectively, in the $K_{L}$ state

$$
\delta=\frac{|p|^{2}-|q|^{2}}{|p|^{2}+|q|^{2}}
$$


The experimenter has no control of the kaon decay, neither of the mode nor of the time. The experimenter can only sort all observed events in proper decay modes and time intervals. We call this procedure opposite to the active measurement described above a passive measurement procedure of strangeness.

The second basis $\left\{K_{S}, K_{L}\right\}$ consists of the short- and long-lived states having well defined masses $m_{S(L)}$ and decay widths $\Gamma_{S(L)}$. It is the appropriate basis to discuss the kaon propagation in free space, because these states preserve their own identity in time. Due to the huge difference in the decay widths the $K_{S}$ 's decay much faster than the $K_{L}$ 's. Thus in order to observe if a propagating kaon is a $K_{S}$ or $K_{L}$ at time $t$, one has to detect at which time it subsequently decays. Kaons which are observed to decay before $\simeq t+4.8 \tau_{S}$ are identified as $K_{S}$ 's, while those surviving after this time are assumed to be $K_{L}$ 's. The number $4.8 \tau_{S}$ is obtained by setting the probability to observe a $K_{S}$ equal to the probability that a $K_{L}$ state does not decay, i.e. $e^{-\Gamma_{S} t}=1-e^{-\Gamma_{L} t} \longrightarrow t \approx 4.8 \tau_{S}$. This means that we equalized the contamination of both samples, that is a few parts per mil (see also Refs. [11, 12]). Note that the experimenter doesn't care about the specific decay mode, he records only a decay event at a certain time. We call this procedure an active measurement of lifetime.

Since the neutral kaon system violates the $C P$ symmetry the mass eigenstates are not strictly orthogonal, $\left\langle K_{S} \mid K_{L}\right\rangle \neq 0$. However, neglecting $C P$ violation - of the order of $10^{-3}$ - the $K_{S}$ 's are identified by a $2 \pi$ final state and $K_{L}$ 's by a $3 \pi$ final state. We call this procedure a passive measurement of lifetime, since the kaon decay times and decay channels used in the measurement are entirely determined by the quantum nature of kaons and cannot be influenced by the experimenter.

Summarizing, we have the following two measurement procedures for the strangeness and the mass-eigenstate bases:

Strangeness basis $\left\langle K^{0} \mid \bar{K}^{0}\right\rangle=0$

\begin{tabular}{|c|c|}
\hline \hline Active measurements (strong interactions) & Passive measurements (semileptonic decays) \\
\hline \hline$K^{0}+p \longrightarrow K^{+}+n$ & $\bar{K}^{0}(\bar{d} s) \longrightarrow \pi^{+}(\bar{d} u) l^{-} \bar{v}_{l}$ \\
$\bar{K}^{0}+p \longrightarrow \Lambda+\pi^{+}$ & $K^{0}(d \bar{s}) \longrightarrow \pi^{-}(d \bar{u}) l^{+} v_{l}$ \\
$\bar{K}^{0}+n \longrightarrow K^{-}+p, \Lambda+\pi^{0}$ & \\
\hline
\end{tabular}

\begin{tabular}{|c|c|}
\multicolumn{2}{|c|}{ Mass eigenstate basis $\left\langle K_{S} \mid K_{L}\right\rangle=\frac{2 \operatorname{Re}\{\varepsilon\}}{1+|\varepsilon|^{2}} \approx 3 \cdot 10^{-3}$} \\
\hline Active measurements (free propagation) & Passive measurements (2 or 3 $\pi$ decay modes) \\
\hline \hline $\begin{array}{c}\rightarrow \text { any decay mode observed before } \\
4.8 \tau_{S} \text { are identified as } K_{S} \text { 's }\end{array}$ & $\rightarrow 2 \pi$ 's are identified as $K_{S}$ 's \\
Misidentification: few parts in $10^{-3} !$ & Misidentification: few parts in $10^{-3}$ ! are identified as $K_{L}$ 's \\
\hline
\end{tabular}




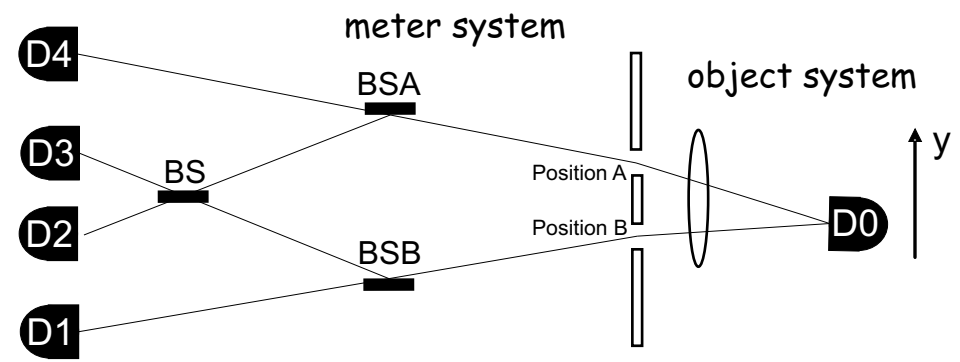

Figure 2: Here the setup of a partially active eraser is sketched. An entangled photon pair can be produced either in region $A$ or in region $B$. If either detector $D 1$ or $D 4$ clicks, one knows the production region $A$ or $B$, i.e. one has full which way information. Clicks of the detectors $D 2$ or $D 3$ erase the information, interference is observed. It is a partially active eraser, because the mark is erased by a probabilistic law, however, the experimenter has still partially control over the erasure, she/he can choose the ratio of transmittivity to reflectivity of the beam splitter $B S A$ and $B S B$.

\section{The active eraser with active measurements}

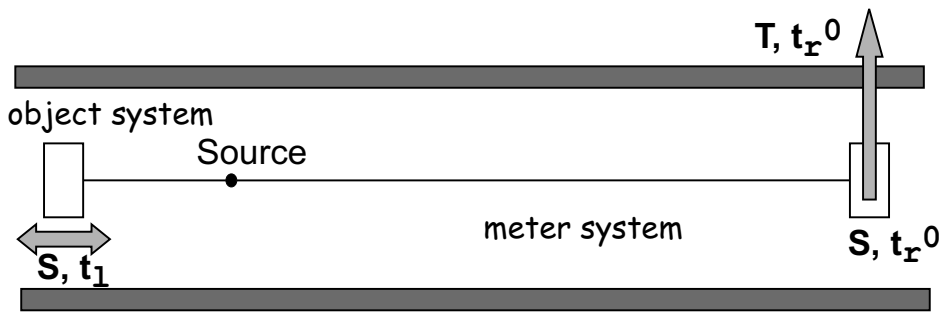

Figure 3: The figure shows the two setups for an eraser with active marking and active measurements. On the object system (left hand side) strangeness is actively measured while on the meter system (right hand side) the experimenter measures actively the strangeness or lifetime by inserting or not the piece of matter.

Let us first discuss the photon analogy, e.g., the two experimental setups in Ref. [4]. In the first setup two interfering two-photon amplitudes are prepared by forcing a pump beam to cross twice the same nonlinear crystal. Idler (meter) and signal (object) photons ${ }^{1}$ from the first down conversion are marked by rotating their polarization by $90^{\circ}$ and then superposed to the idler (i) and signal (s) photons emerging from the second passage of the beam through the crystal. If type-II spontaneous parametric down conversion were used, we had the state ${ }^{2}$

$$
|\psi\rangle=\frac{1}{\sqrt{2}}\{\underbrace{|V\rangle_{i} \otimes|H\rangle_{s}}_{\text {second passage }}-e^{i \Delta \phi} \underbrace{|H\rangle_{i} \otimes|V\rangle_{s}}_{\text {first passage }}\},
$$

where the relative phase $\Delta \phi$ is under control by the experimenter. The signal photon, the object system, is always measured after crossing a polarization analyzer aligned at $+45^{0}$, see Fig. 1. Due to entanglement, the vertical or horizontal idler polarization supplies full which way information for the signal (object) system, i.e., whether it was produced at the first or second passage. No

\footnotetext{
${ }^{1}$ For historical reasons for photons idler/signal is used rather than meter/object.

${ }^{2}$ The authors of Ref. [4] used type-I crystals in their experiment.
} 
interference can be observed in the signal-idler joint detections. To erase this information, the idler photon has to be detected in the $+45^{\circ} /-45^{\circ}$ basis.

In case of entangled kaons the state is described by

$$
\begin{aligned}
|\psi(t=0)\rangle & =\frac{1}{\sqrt{2}}\left\{\left|K^{0}\right\rangle_{l} \otimes\left|\bar{K}^{0}\right\rangle_{r}-\left|\bar{K}^{0}\right\rangle_{l} \otimes\left|K^{0}\right\rangle_{r}\right\}, \\
& =\frac{1}{\sqrt{2}}\left\{\left|K_{S}\right\rangle_{l} \otimes\left|K_{L}\right\rangle_{r}-\left|K_{L}\right\rangle_{l} \otimes\left|K_{S}\right\rangle_{r}\right\},
\end{aligned}
$$

the last equality is obtained by neglecting the $\mathscr{C} \mathscr{P}$ violation. The analogy with Eq. (3.1) is quite obvious, however, kaons evolve in time, such that the state depends on the two time measurements on the left hand side, $t_{l}$, and on the right hand side, $t_{r}$, or more precisely on $\Delta t=t_{l}-t_{r}$, when normalized $^{3}$ to surviving kaon pairs $(\otimes$ is from now on suppressed)

$$
\begin{aligned}
|\psi(\Delta t)\rangle=\frac{1}{\sqrt{1+e^{\Delta \Gamma \Delta t}}} & \left\{\left|K_{L}\right\rangle_{l}\left|K_{S}\right\rangle_{r}-e^{i \Delta m \Delta t} e^{\frac{1}{2} \Delta \Gamma \Delta t}\left|K_{S}\right\rangle_{l}\left|K_{L}\right\rangle_{r}\right\} \\
=\frac{1}{2 \sqrt{1+e^{\Delta \Gamma \Delta t}}} & \left\{\left(1-e^{i \Delta m \Delta t} e^{\frac{1}{2} \Delta \Gamma \Delta t}\right)\left\{\left|K^{0}\right\rangle_{l}\left|K^{0}\right\rangle_{r}-\left|\bar{K}^{0}\right\rangle_{l}\left|\bar{K}^{0}\right\rangle_{r}\right\}\right. \\
& \left.+\left(1+e^{i \Delta m \Delta t} e^{\frac{1}{2} \Delta \Gamma \Delta t}\right)\left\{\left|K^{0}\right\rangle_{l}\left|\bar{K}^{0}\right\rangle_{r}-\left|\bar{K}^{0}\right\rangle_{l}\left|K^{0}\right\rangle_{r}\right\}\right\} .
\end{aligned}
$$

We notice that the phase $\Delta m \Delta t$ introduces automatically a time dependent relative phase between the two amplitudes. The marking and erasure operations can be performed on entangled kaon pairs as in the optical case discussed above. The object kaon flying to the left hand side is measured always actively in the strangeness basis, see Fig. 3. As in the optical version the kaon flying to the right hand side, the meter kaon, is measured actively either in the strangeness basis by placing a piece of matter in the beam or in the lifetime basis by removing the piece of matter. Both measurements are actively performed. In the latter case we obtain information about the lifetime, namely which width the object kaon has, and clearly no interference in the joint detections can be observed.

\footnotetext{
${ }^{3}$ Thanks to this normalization, we work with bipartite two-level quantum systems like polarization entangled photons or entangled spin-1/2 particles. For an accurate description of the time evolution of kaons and its implementation consult Ref. [13, 14].
} 


\section{The partially passive quantum eraser with active measurements}

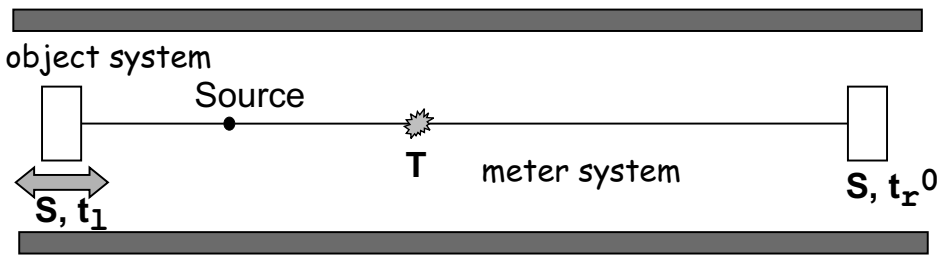

Figure 4: The figure shows the setup for a partially passive quantum marking and active measurements on both sides. On the object system (left hand side) strangeness is actively measured while on the meter system (right hand side) the strangeness or lifetime is actively measured. The meter system "decides" whether the "wave-like" property or the "particle-like" property is observed.

In Fig. 2, a setup is sketched where either at position A or B an entangled photon pair is produced, which was realized in Ref. [5]. "Clicks" on detector D1 or D4 provide "which way" information. "Clicks" on detector D2 and D3 give no information about the position A or B, interference is observed in the joint events of the two photons, see Fig. 2.

In the kaon case, a piece of matter is permanently inserted into both beams where the one for the meter system at the right hand side is fixed at time $t_{r}^{0}$, see Fig. 4. The experiment observes the region from the source to the piece of matter at the right hand side. In this way the kaon moving to the right - the meter system - takes the choice to show "which width" information by its decay during its free propagation until $t_{r}^{0}$ or not by being absorbed in the piece of matter. Again strangeness or lifetime is measured actively. The choice whether the "wave-like" property or the "particle-like" property is observed is naturally given by the instability of the kaons. It is "partially active", because the experimenter can choose at which fixed time $t_{r}^{0}$ the piece of matter is inserted. This is analogous to the optical case where the experimenter can choose the transmittivity of the two beam-splitters $B S A$ and $B S B$ in Fig. 2 .

Furthermore, note that it is not necessary to identify $K_{S}$ versus $K_{L}$ for demonstrating the quantum marking and eraser principle.

\section{The passive eraser with "passive" measurements on the meter}

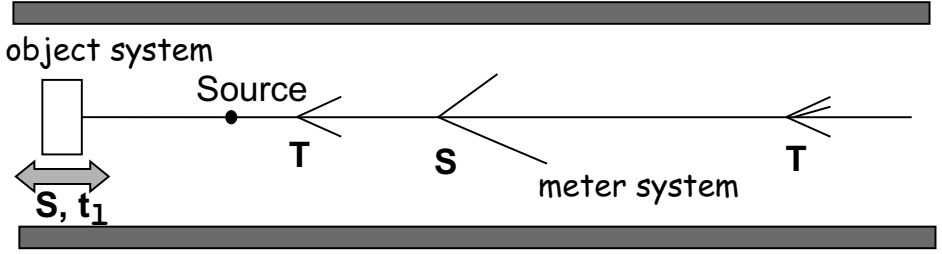

Figure 5: The figure shows a setup of a quantum eraser which has no photon analog. On the object system (left hand side) strangeness is actively measured while on the meter system (right hand side) the strangeness or lifetime is observed by its decay (passive measurement). The experimenter has no control whether the lifetime mark is read out or not. 
Next we consider the setup in Fig. 5. We take advantage - and this is specific for kaons- of the passive measurement. Again the strangeness content of the object system - kaon moving to the left hand side - is actively measured by inserting a piece of matter into the beam. In the beam of the meter no matter is placed in, the kaon moving to the right propagates freely in space. This corresponds to a passive measurement of either strangeness or lifetime on the meter by recording the different decay modes of neutral kaons. If a semileptonic decay mode is found, the strangeness content is measured. In the joint events interference is observed. If a two or three $\pi$ decay is observed, the lifetime is measured and thus "which width" information of the object system is obtained, no interference is seen in the joint events. Clearly we have a completely passive erasing operation on the meter, the experimenter has no control whether the lifetime mark is read out or not.

This experiment has no analog to any other considered two-level quantum system.

\section{The passive eraser with "passive" measurements}

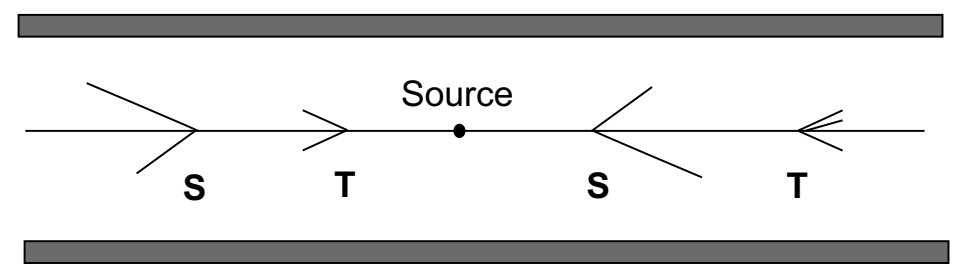

Figure 6: For this type of quantum eraser, it is not clear which side plays the meter/object role as it is totally symmetric and it involves only passive measurements. This clearly has no analog to photon experiments.

Finally we mention the setup in Fig. 6, where both kaons evolve freely in space and the experimenter observes passively their decay modes and times. The experimenter has no control over individual pairs neither on which of the two complementary observables at each kaon is measured nor when it is measured. This setup is totally symmetric, thus it is not clear which side plays the role of the meter. In this sense, one could claim that this experiment should not be considered as a quantum eraser. But one could also claim that this experiment reveals the true essence of the erasure phenomenon: Until the two measurements (one in each side) are completed, the factual situation is undefined; once one has the measurement results on both sides, the whole set of joint events can be classified in two subsets according to the kind of information (on strangeness or on lifetime) that has been obtained. The lifetime subset shows no interference, whereas fringes and antifringes appear when sorting the strangeness subset events according to the outcome, $K^{0}$ or $\bar{K}^{0}$, of the meter kaon.

Summarizing, it is remarkable that for all four presented setups combining active and passive measurement procedures lead to the same observable probabilities! And this is even true regardless of the temporal ordering of the measurements!

\section{Conclusions}

We have discussed the possibilities offered by neutral kaon states, such as those copiously 
produced by $\Phi$-resonance decays at the DA $\Phi$ NE machine, to investigate fundamental issues of quantum mechanics: the quantum eraser phenomena. Neutral kaons seem to be the natural system to study these phenomena because it allows for a clear conceptual simplification and one obtains the relevant formulae in a transparent and non-controversial way.

Moreover, the possibility of performing passive measurements, a specific feature of neutral kaons not shared by other systems, has been shown to open new options for the quantum eraser. Demonstrating the very concept of a quantum eraser: sorting events according to the information available.

The CPLEAR experiment [15] did only part of the job (active strangeness-strangeness measurements) with every limited statistics and absorber positions. The KLOE-2 experiment could do the full program: Monte Carlo studies for both active and passive measurements are in progress, and concrete designs for positioning thin absorbers close to the interaction point - requiring a modification of the beam pipe - are under investigations.

\section{References}

[1] M. Arndt, O. Nairz, J. Vos-Andreae, C. Keller, G. Van der Zouw and A. Zeilinger, Nature 401, 680 (1999).

[2] M. O. Scully and K. Drühl, Phys. Rev. A 25, 2208 (1982).

[3] S. Dürr and G. Rempe, Opt. Commun. 179, 323 (2000).

[4] T.J. Herzog, P.G. Kwiat, H. Weinfurter and A. Zeilinger, Phys. Rev. Lett 75, 3034 (1995).

[5] Y.-H. Kim, R. Yu, S.P. Kuklik, Y. Shih and M.O. Scully, Phys. Rev. Lett. 84, 1 (2000).

[6] T. Tsegaye, G. Björk, M. Atatüre, A.V. Sergienko, B.W.A. Saleh and M.C. Teich, Phys. Rev. A 62, 032106 (2000).

[7] S.P. Walborn, M.O. Terra Cunha, S. Padua and C.H. Monken, Phys. Rev. A 65, 033818 (2002).

[8] A. Trifonov, G. Björk, J. Söderholm and T. Tsegaye, Eur. Phys. J. D 18, 251 (2002).

[9] H. Kim, J. Ko and T. Kim, Phys. Rev. A 67, 054102 (2003).

[10] Y. Aharonov and M.S. Zubairy, Science 307, 875 (2005).

[11] A. Bramon, G. Garbarino and B. C. Hiesmayr, Phys. Rev. Lett. 92, 020405 (2004).

[12] A. Bramon, G. Garbarino and B. C. Hiesmayr, Phys. Rev. A 68, 062111 (2004).

[13] B. C. Hiesmayr, European Physical Journal C, Vol. 50, 73 (2007).

[14] R. A. Bertlmann, B. C. Hiesmayr, Phys. Rev. A 63, 062112 (2001).

[15] A. Apstolakis et.al., Phys. Lett. B 422, 339 (1998). 